\title{
Myxozoa + Polypodium - a common route to endoparasitism
}

\author{
Beth Okamura ${ }^{1, *}$ and Alexander Gruhl ${ }^{1,2}$ \\ ${ }^{1}$ Natural History Museum, Department of Life Sciences, London, United Kingdom \\ ${ }^{2}$ Max Planck Institute for Marine Microbiology, Bremen, Germany \\ *b.okamura@nhm.ac.uk
}

\section{Keywords}

Cnidaria, phylogeny, fish parasites, evolution of parasitism

\section{Summary}

Recent evidence places the problematic Polypodium, a parasite of fish eggs, firmly as sister taxon to Myxozoa within the Cnidaria. This resolution suggests a single route to endoparasitism in Cnidaria, with larval stages of a common ancestor exploiting fish as first hosts, and enables new interpretations and insights regarding evolutionary transitions associated with endoparasitism.

\section{Main Text}

Myxozoans were first encountered in 1825 as endoparasitic organisms that caused cysts in whitefish. They were subsequently found in a diversity of marine and freshwater fish and, more recently, in other vertebrates (amphibians, reptiles, birds and shrews). The complex two-host life cycle of myxozoans was recognised when organisms previously regarded as belonging to separate taxa (Actinosporea and Myxosporea) were demonstrated as different stages in a common life cycle involving invertebrate final and fish intermediate hosts. Actinosporea and Myxosporea were thus united in the Myxosporea, sister now to the recently discovered Malacosporea. Invertebrate hosts identified so far comprise annelids in the derived Myxosporea and freshwater bryozoans in the early diverging Malacosporea. Because of the extremely simple morphology of myxozoan trophic stages, myxozoans were long classified as protists despite relatively early appreciation that infectious spores are multicellular. Their higher level relationships remained enigmatic and contentious because rapid molecular evolution and associated long branch attraction greatly obscured placement until phylogenomic investigations finally revealed myxozoans to group within Cnidaria with strong support as sister to Medusozoa. This is further supported by correspondences in fine structure and protein composition of myxozoan cell organelles-the polar capsules-and cnidarian nematocysts. Earlier phylogenetic investigations based on SSU rDNA data, however, had hinted at an affinity of myxozoans and Polypodium hydriforme with the inclusion of Polypodium often resolving Myxozoa + Polypodium as sister to Bilateria (Figure 1A). Like myxozoans, Polypodium is an endoparasite of fish, in this case infecting eggs of acipenseriforms (sturgeon and paddlefish). Unlike myxozoans, Polypodium has retained a cnidarian-like free-living stage whose morphology and development suggested placement within the Medusozoa (jellyfish, Hydra and their relatives).

Chang et al. [1] have addressed the outstanding issue of the relationship between myxozoans and Polypodium. By analysing genomic and transcriptomic data, they confirm a clade comprising Polypodium + Myxozoa that is sister to Medusozoa (Figure 1B) and provide evidence for extremely 
reduced genome size and gene content in the myxozoan Kudoa iwatai. These results have implications for how we may now view evolution within Cnidaria and provide insights into evolutionary transitions associated with endoparasitism. Below we elaborate on some of these issues.

Myxozoans and Polypodium share peculiar cytological and developmental features. In Polypodium early stage binucleate cells invade fish eggs [2]. Larval development ensues when one cell becomes enclosed by the other, producing a cell-within-cell arrangement. Early stages in myxozoans include binucleate cells in invertebrate hosts and primary cells containing secondary cells (a cell-within-cell arrangement) in fish hosts [3]. In Polypodium the outer cell (trophamnion) phagocytoses yolk that nourishes the inner cell which divides and develops into a planuliform larva. Myxozoan trophic stages in fish occur as pseudoplasmodia (large uninucleate cells) or as syncytial plasmodia (large multinucleate cells; Figure $1 \mathrm{C}$ ) within which infectious multicellular spores develop. Similarly enclosed internal cells develop in parasitic protists (species of Paramyxa and Paramarteilia) suggesting that protists and metazoans have independently converged to provision and protect parasitic stages at the cellular level.

The lack of developmental genes (e.g. Hox-like, Wnt, Runx and Hedgehog genes) in the genomes and transcriptomes of the two representative myxosporeans ( $K$. iwatai and Myxobolus cerebralis) suggests a loss of genes no longer required in highly morphologically simplified, exclusively plasmodia-forming myxosporeans. However, it is premature for Chang et al. to conclude that such gene loss characterises all myxozoans. Evidence for this would require analyses of similar data for the early diverging Malacosporea - a group morphologically much more complex than myxosporeans, occurring as trophic stages (sacs and worms) with epithelial tissues and, in some cases, muscles (in the worm-like Buddenbrockia; Figure 1C) in invertebrate hosts. Furthermore, the genome of Thelohanellus kitauei demonstrates streamlining and loss of genes associated with metabolism [4] but remains eightfold larger, begging the question of why gene loss and compaction is so variable between myxosporeans that both occur as intercellular plasmodia within fish tissues. Possible answers include genome reduction due to genetic drift in isolated populations with small effective population size (for example possible bottlenecking of $K$. iwatai in the Red Sea), shifts in mutation rate, correlations with cell size or metabolic activity, and reductions in number and activity of transposable elements or in intergenic spacers and introns $[5,6]$.

The sister taxon relationship of Myxozoa and Polypodium suggests that their common ancestor exploited fish as first hosts (since Polypodium appears to utilise only fish) and that fish were invaded by larval stages [7]. In turn, this suggests the subsequent adoption of invertebrate hosts by the myxozoan adult stage - a scenario supported by features including meiosis and, in worm-like Buddenbrockia, longitudinal muscles in a tetraradial arrangement (Figure 1C) [8]. Tetradial symmetry is shared with adult stages of Medusozoa and the problematic Staurozoa. Gene expression studies offer an excellent opportunity to confirm these inferences.

A recent molecular dating study (based on SSU rDNA analysis) has inferred that the marine myxosporean clade diverged near the time that cartilaginous fish originated in the Silurian [9], thus implying an earlier divergence for Polypodium + Myxozoa. However, a study (based on nine unlinked proteins) of major ray-finned fish lineages [10] infers a Devonian origin for the Acipensiformes. As acipenseriform fishes are present-day Polypodium hosts this suggests an earliest possible Devonian 
divergence of Polypodium + Myxozoa and hence a stem lineage that may have exploited early primitive fish and which persisted for a long period (the Medusozoa originated in the Precambrian). Potential host shifts and undersampling, however, make these scenarios highly speculative. For example, Polypodium could have shifted more recently from teleost to early diverging acipenseriform hosts, or basal Polypodium lineages infecting teleosts may be undetected. Because myxozoans have no fossil record and may have undergone many host switches over evolutionary time, inferences about origins and times of diversification remain problematic. Nevertheless, we can expect that future research will shed further light on the transition to endoparasitism and subsequent diversification in this fascinating and substantial radiation of cnidarians. The common adoption of fish hosts may also be significant to the aquaculture and caviar industries - for example in the design of broad spectrum drugs or vaccines that target molecular features, such as virulence factors, that are shared by Polypodium and Myxozoa.

Figure 1. A) Long branch attraction and varying analytical approaches often inferred a sister taxon relationship of Myxozoa to Bilateria, including when Polypodium hydriforme was incorporated in SSU rDNA molecular phylogenetic analyses. Animal captions (except Myxozoa, Polypodium, Medusozoa) from www.phylpic.org. Creative Commons Attribution-ShareAlike 3.0 Unported license. B) Analyses of transcriptomes and genomes enable Chang et al. (2015) to infer $P$. hydriforme + Myxozoa as sister to Medusozoa. C) Myxozoa is comprised of the Malacosporea, whose spore-forming trophic stages develop epithelial layers (and tetraradial muscles in the worm-like Buddenbrockia plumatellae as depicted in a schematic cross-section here), and the Myxosporea, whose spore-forming trophic stages lack tissues and develop exclusively as multinucleate plasmodia (depicted schematically here) or pseudoplasmodia. cc-connecting cell, cy-cytoplasm, ep-outer epithelium, mu-muscle block, nu - nucleus, sp-spore

\section{References}

[1] Chang, E.S. et al. (2015) Genomic insights into the evolutionary origin of Myxozoa within Cnidaria. Proc. Natl. Acad. Sci. USA 112, 14912-14917, doi:10.1073/pnas.1511468112

[2] Raikova, E.V. (1994) Life cycle, cytology, and morphology of Polypodium hydriforme, a coelenterate parasite of the eggs of Acipenseriform fishes. J. Parasitol. 80, 1-22

[3] Feist, S.W. et al. (2015) Cellular Processes in Myxozoans. In Myxozoan evolution, ecology and development (Okamura, B., Gruhl, A., Bartholomew, J.L., eds), pp. 139-154, Springer International Publishing

[4] Yang, Y. et al. (2014) The genome of the myxosporean Thelohanellus kitauei shows adaptations to nutrient acquisition within its fish host. Genome Biol. Evol. 6, 3182-3198, doi:10.1093/gbe/evu247

[5] Wernegreen, J.J. (2015) Endosymbiont evolution: predictions from theory and surprises from genomes. Ann. N.Y. Acad. Sci. 1360, 16-35

[6] Poulin, R. and Randhawa, H.S. (2015) Evolution of parasitism along convergent lines: from ecology to genomes. Parasitol. 142, S6-S15 
[7] Okamura, B. and Gruhl, A. (2015) Myxozoan affinities and route to endoparasitism. In Myxozoan evolution, ecology and development (Okamura, B., Gruhl, A., Bartholomew, J.L., eds), pp. 23-44, Springer International Publishing

[8] Gruhl, A. and Okamura, B. (2012) Development and myogenesis of the vermiform Buddenbrockia (Myxozoa) and implications for cnidarian body plan evolution. EvoDevo 3,10

[9] Kodádková, A. et al. (2015) Bipteria vetusta n. sp. - an old parasite in an old host: tracing the origin of myxosporean parasitism in vertebrates. Int. J. Parasitol. 45, 269-276

[10] Near, T.J. et al. (2012) Resolution of ray-finned fish phylogeny and timing of diversification. Proc. Natl. Acad. Sci. USA 109, 13698-13703 
Bilateria

Myxozoa

Polypodium

Medusozoa

Cnidaria

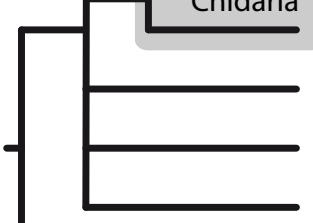

Anthozoa

Ctenophora

Placozoa

Porifera

Choanoflag.

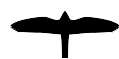

(B)
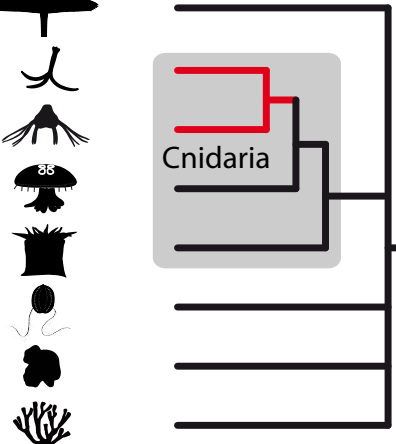

0
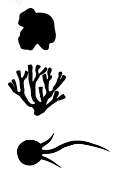

\section{(C) Myxozoa}

Malaco- Myxosporea sporea

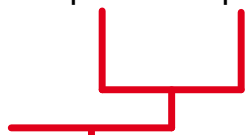

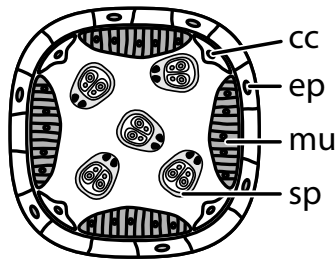

Malacosporea

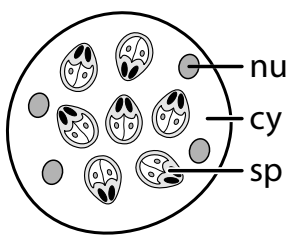

Myxosporea 